\title{
Effect of High Dose Methylprednisolone Pulse Therapy Followed by Oral Prednisolone Administration on the Production of Anti-TSH Receptor Antibodies and Clinical Outcome in Graves' Disease
}

\author{
SUMIHISA KUBOTA, HIDEMI OHYE, EIJUN NISHIHARA, TAKUMI KUDO, MITSURU ITO, SHUJI FUKATA, \\ NOBUYUKI AMINO, KANJI KUMA AND AKIRA MIYAUCHI
}

Kuma Hospital, Kobe 650-0011, Japan

\begin{abstract}
Little is known about the immunosuppressive effect of glucocorticoids on TSH receptor antibodies. We observed the long-term prognosis and serum TSH binding inhibitor immunoglobulin (TBII) levels in patients with Graves' ophthalmopathy who had received intravenous methylprednisolone pulse therapy (pulse therapy) followed by oral prednisolone administration in order to ascertain how long the immunosuppressive effect of glucocorticoids continued. This is the first report on the effect of pulse therapy on Graves' disease outcome. We observed 67 patients who were treated by antithyroid drugs (ATD) alone for 2 years after pulse therapy. TBII was evaluated before and 3, 6, 12, 18, and 24 months after pulse therapy. The mean TBII decreased significantly 3 months after pulse therapy $(\mathrm{p}<0.001)$, and was maintained until 24 months. There were 24 patients whose TBII was positive $(>15 \%)$ at 24 months, in whom the mean TBII decreased significantly 3 to 6 months after pulse therapy $(p<0.001)$, but increased again at 12 to 24 months ( $<<0.05)$. Thus, the immunosuppressive effect of glucocorticoids may be lost at 12 months after pulse therapy in these patients. The remission rate in the pulse therapy group was $40.98 \%$, and that of the control patient group was $48.57 \%$. There was no significant difference between the two. These results suggest that the immunosuppressive effect of pulse therapy was temporary, and that pulse therapy did not increase remission rate of Graves' disease.
\end{abstract}

Key words: Intravenous methylprednisolone pulse therapy, Graves' disease, TRAb, Ophthalmopathy, Remission

(Endocrine Journal 52: 735-741, 2005)

IT is well established that Graves' thyrotoxicosis is induced by thyroid stimulating antibodies which are produced by autoimmunity to the TSH receptor. In general, glucocorticoids are frequently used to treat autoimmune diseases. However, glucocorticoids are seldom used for the treatment of Graves' disease, since there are other effective treatments with less adverse effects like antithyroid drugs (ATD). Therefore the effect of glucocorticoid therapy is poorly evaluated in Graves' disease [1-3]. Exceptionally large amounts of glucocorticoids are used for Graves' patients with se-

Received: July 8, 2005

Accepted: September 12, 2005

Correspondence to: Dr. Sumihisa KUBOTA, Kuma Hospital, 82-35 Shimoyamate-Dori, Chuo-Ku, Kobe 650-0011, Japan vere ophthalmopathy. In these cases, the high dose of intravenous methylprednisolone pulse therapy (pulse therapy) often improves ophthalmic symptoms [4-15].

Glucocorticoids induce both $\mathrm{T}$ and $\mathrm{B}$ lymphocytes apoptosis, inhibition of immunoglobulin synthesis, and inhibition of the action of NF- $\mathrm{BB}$ which plays a crucial role in inducing cytokine gene transcription [16, 17]. As a result, glucocorticoids have an immunosuppressive effect and antiinflammatory effect.

Pulse therapy possibly affects the production of antiTSH receptor antibodies (TRAb) and favorably changes the clinical course in Graves' disease. Thus, a positive outcome would be expected in the patients who were treated with pulse therapy. As far as we know, however, the effect of pulse therapy on disease outcome has not been confirmed. Therefore we observed the effect 
of pulse therapy followed by oral prednisolone administration on the TRAb production and remission rate in patients with Graves' disease associated with active ophthalmopathy. These parameters were compared with patients with Graves' disease not undergoing pulse therapy.

In addition, there are several reports that suggest an association between IgE and remission of Graves' disease. We also measured $\operatorname{IgE}$ before pulse therapy and examined the association between prognosis of Graves' disease and IgE [18-20].

\section{Subjects and Methods}

\section{Subjects}

We treated 113 patients with Graves' ophthalmopathy by pulse therapy between 1995 and 2000. All patients had active and severe ophthalmopathy. Of these, 86 patients were treated also with ATD in order to control thyroid function. We were able to follow up $61 \mathrm{pa}-$ tients (41 female, 20 male), aged $51.11 \pm 10.17 \mathrm{yr}$ (mean $\pm \mathrm{SD}$ ) for 2 years after pulse therapy.

We selected 132 consecutive patients with Graves' disease who visited our hospital in 1998. One hundred nine of 132 patients were treated with ATD alone. Of these, we could follow up 70 patients (56 female, 14 male), aged $34.87 \pm 14.38 \mathrm{yr}$ (mean $\pm \mathrm{SD}$ ) for more than 3 years.

There was no difference in sex, thyroid gland volume estimated by ultrasound, pretreatment TBII values, or pretreatment FT4 and FT3 levels between the 2 groups. The mean age of the pulse therapy group was higher than that of the control group. It is for this reason that Graves' ophthalmopathy tends to be more severe in older patients.

\section{Clinical protocol}

One gram of methylprednisolone was infused intravenously daily for 3 successive days, and the patients were observed for 4 days. After 3 cycles of this course, prednisolone $(15 \mathrm{mg} /$ day $)$ was administered orally for 3 months. Fifty-nine of the 61 patients received orbital radiotherapy (overall dose of $20 \mathrm{~Gy}$ in 10 fractions) after the pulse therapy. The remaining 2 patients were treated by pulse therapy alone.

Hyperthyroidism and ophthalmopathy occurred al- most concurrently in 34 patients. Hyperthyroidism preceded ophthalmopathy (median 8.0 months, range 1-60 months) in 27 patients. All patients were treated with the lowest possible dose of ATD to maintain euthyroid. We measured serum level of TBII before and 3 months, 6 months, 12 months, 18 months, and 24 months after the pulse therapy in subjects. Serum level of TBII was also measured before and 3 months, 6 months, 12 months, 18 months, and 24 months after starting ATD therapy in controls. IgE concentrations were measured in 45 patients before pulse therapy. We determined the patients with negative TBII after 2 years ATD therapy to be the ones who achieved remission of Graves' disease, and recommended withdrawal of ATD for them. Some patients rejected our recommendation because of the fear of exacerbation of their ophthalmopathy. The patients who were euthyroid for more than 1 year without ATD were regarded as experiencing remission of Graves' disease in this study.

\section{Ocular evaluation}

Ocular evaluation was performed by an ophthalmologist mainly in eye muscle function. We evaluated the effects of pulse therapy according to the criteria presented by Donaldson et al. [21].

\section{Biochemical analyses}

TBII was measured by radioreceptor assay using TSH receptor antibody assay kit (RSR Ltd., Cardiff, UK). Normal range was less than $15 \%$. TSH, FT4 and FT3 concentrations in sera were measured by enzyme immunoassays (AxSYM TSH, AxSYM FT4, and AxSYM FT3, respectively, Abbott Japan Co., Tokyo, Japan). IgE concentrations were measured by enzyme immunoassays (IMx IgE, Abbott Japan Co., Tokyo, Japan).

\section{Statistical analysis}

Changes in TBII were evaluated by the KruskalWallis nonparametric ANOVA test. Differences between TBII for each month were evaluated by the Mann-Whitney test. Difference between remission rates was evaluated by the $\chi^{2}$-test. To assess whether the TBII factor has predictive value for the outcome of Graves' disease, we used logistic regression analysis. 


\section{Results}

\section{Changes in TBII}

Fig. 1 shows the TBII changes in 61 subject patients and 70 control patients. The mean TBII level decreased significantly 3 months after pulse therapy $(\mathrm{p}<0.001)$, and was maintained until 24 months in the subjects. On the other hand, the mean TBII value in control patients decreased constantly after starting ATD. TBII values at 3 months and 6 months for the pulse therapy group were significantly lower than the values of the control group.

The TBII changes in 24 patients whose TBII was positive $(\geq 15 \%)$ at 24 months after pulse therapy are shown as open circles in Fig. 2. The mean TBII value

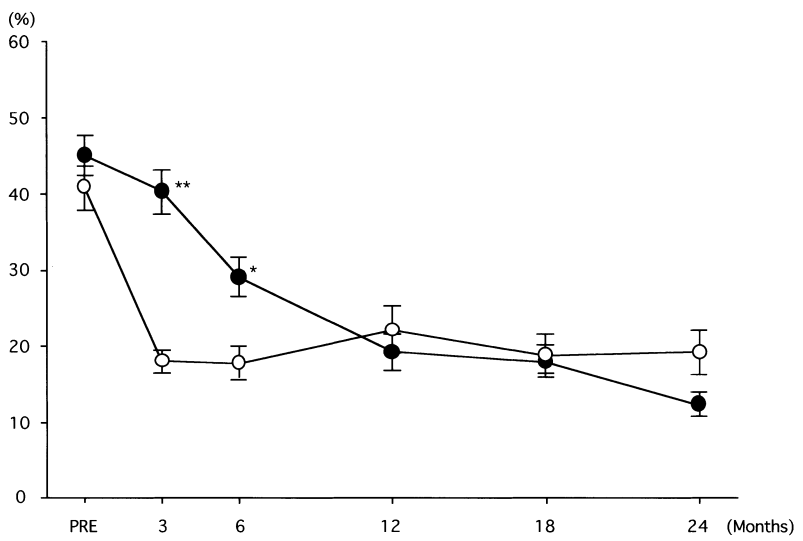

Fig. 1. TBII changes after pulse therapy in 64 patients with Graves' ophthalmopathy (open circle) and in 70 control patients (closed circle). The mean and median TBII levels decreased significantly 3 months after pulse therapy $(\mathrm{p}<0.001)$, and were maintained until 24 months. ** $p<0.001$ compared to controls, ${ }^{*} \mathrm{p}<0.01$ compared to controls decreased significantly 3 to 6 months after pulse therapy $(\mathrm{p}<0.001)$, but increased again at 12 to 24 months $(\mathrm{p}<0.05)$. On the other hand, the mean TBII value in control patients whose TBII was positive $(\geq 15 \%)$ at 24 months decreased steadily after starting ATD $(p<0.001)$. TBII values after 12 months in both groups were quite similar. The immunosuppressive effect of glucocorticoids may be lost at 12 months after pulse therapy in these patients.

Fig. 3 shows the TBII changes in both groups whose TBII was negative $(<15 \%)$ at 24 months. TBII values in the pulse therapy group decreased faster than the control group, but the values showed no difference between the 2 groups after 12 months.

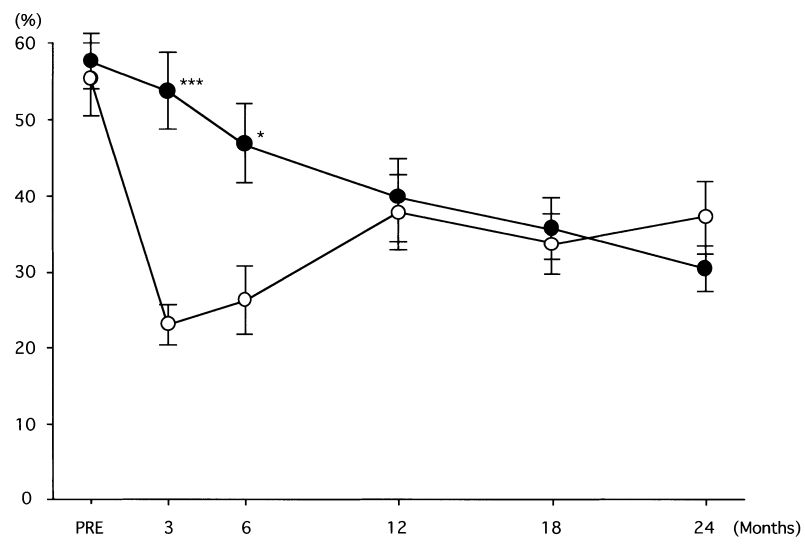

Fig. 2. TBII changes in 24 patients with Graves' disease (open circle) and in control patients (closed circle) whose TBII was positive at 24 months. The mean TBII value decreased significantly 3 to 6 months after pulse therapy $(\mathrm{p}<0.001)$, but increased again at 12 to 24 months $(\mathrm{p}<0.05)$.

*** $\mathrm{p}<0.0001$ compared to controls, ${ }^{*} \mathrm{p}<0.01$ compared to controls

Table 1. Characteristics of subjects and controls

\begin{tabular}{lcc}
\hline & pulse therapy group & control \\
\hline Sex (F/M) & $41 / 20$ & $56 / 14$ \\
Age (yr) & $51.11 \pm 10.17^{\mathrm{a}}$ & $34.87 \pm 14.38$ \\
Thyroid gland (g) & $30.48 \pm 21.97$ & $34.78 \pm 23.51$ \\
TBII (pretreatment) (\%) & $40.42 \pm 23.63$ & $45.15 \pm 22.17$ \\
FT4 (pretreatment of ATD) $(\mathrm{ng} / \mathrm{dl})$ & $3.97 \pm 1.44$ & $4.54 \pm 1.29$ \\
FT3 (pretreatment of ATD) $(\mathrm{pg} / \mathrm{ml})$ & $13.19 \pm 7.94$ & $16.35 \pm 7.47$ \\
FT4 (pretreatment of pulse thrapy) $(\mathrm{ng} / \mathrm{dl})$ & $1.27 \pm 0.83$ & \\
FT3 (pretreatment of pulse therapy) $(\mathrm{pg} / \mathrm{ml})$ & $3.86 \pm 3.26$ & \\
\hline
\end{tabular}

Values are means \pm SEM. ${ }^{\text {a }} \mathrm{P}<0.0001$ 


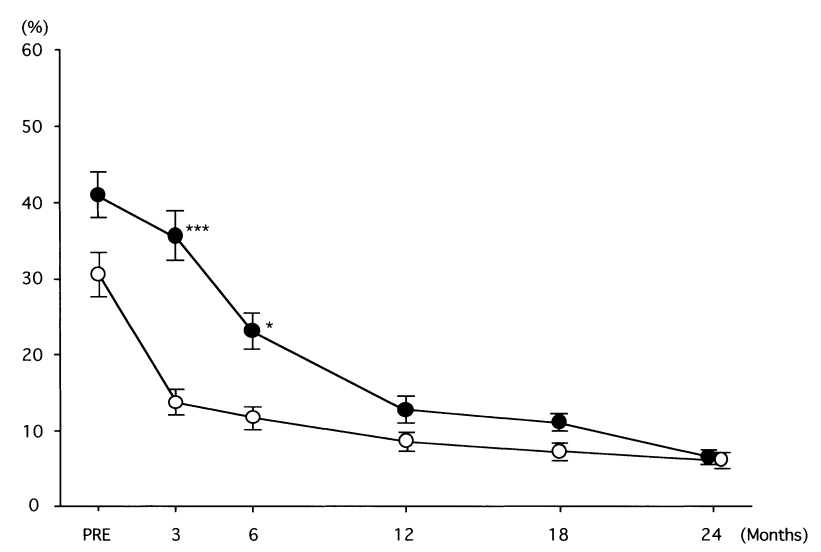

Fig. 3. TBII changes in 24 patients with Graves' disease (open circle) and in control patients (closed circle) whose TBII was negative at 24 months. The mean TBII value decreased significantly 3 months after pulse therapy $(\mathrm{p}<0.001)$.

$* * * \mathrm{p}<0.0001$ compared to controls, $* \mathrm{p}<0.01$ compared to controls

\section{Remission rate}

We evaluated the remission rate of Graves' hyperthyroidism in the patients who were treated with pulse therapy and ATD. The patients who were treated with radioiodine therapy, surgery, or who dropped out, were excluded. The remission rate of the pulse therapy group was $40.98 \%(25 / 61)$, and that of the control group was $48.57 \%$ (34/70). There was no significant difference between the 2 groups.

\section{Effect of pulse therapy for ophthalmopathy}

The outcome of pulse therapy was excellent in 3 (4.5\%), good in $39(58.2 \%)$, fair in $20(29.9 \%)$, no response in $5(7.4 \%)$, and worse in 0 out of 67 patients who were treated by ATD alone. There was no relationship between the outcome and TBII values 24 months after pulse therapy (logistic regression analysis: $\mathrm{p}($ Prob $>$ ChiSq $)=0.5485)$. The remission of Graves' disease was achieved by 3 patients in excellent $(100 \%)$, 12 in $\operatorname{good}(30.8 \%), 10$ in fair $(50 \%)$, and 2 in no response $(40 \%)$. Effectiveness of pulse therapy in the patients of ophthalmopathy also do not correlate with the remission rate of Graves' disease.

\section{IgE concentrations}

The results of IgE measurement appear in Fig. 4.

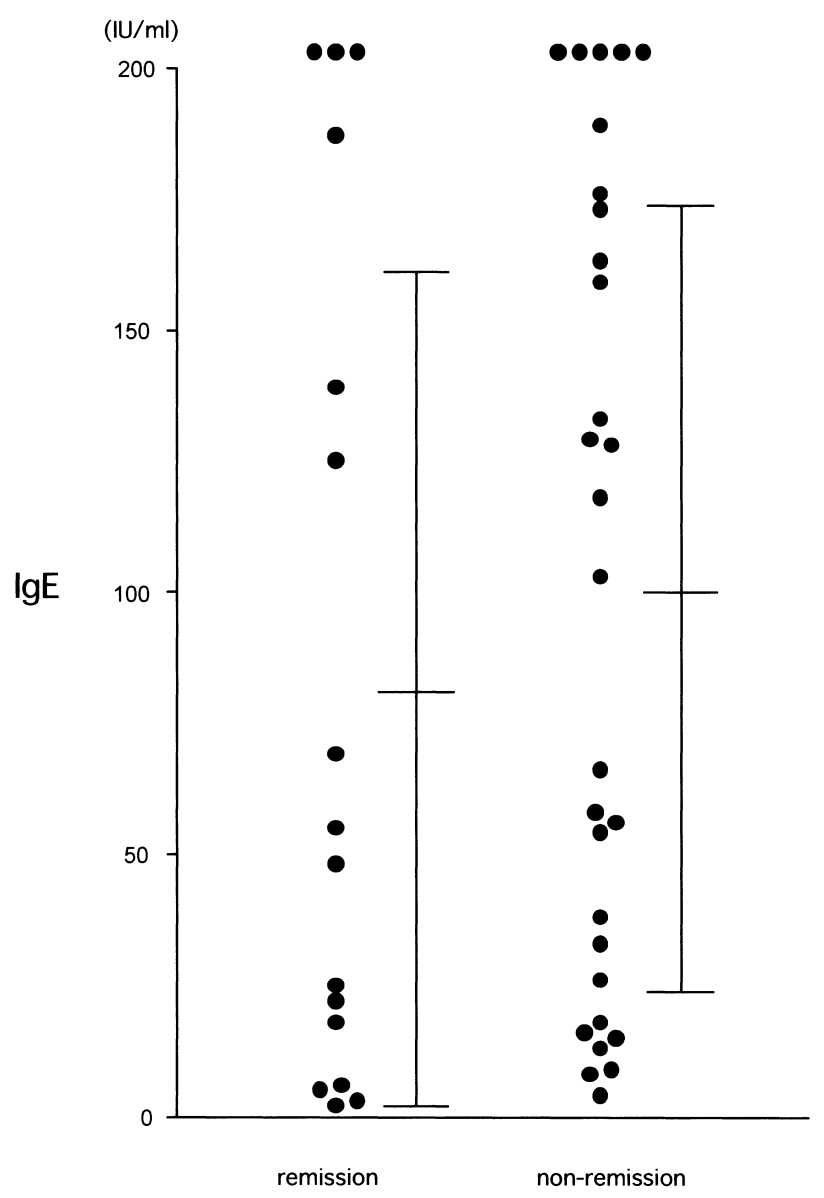

Fig. 4. Serum $\operatorname{IgE}$ concentrations in remission group $(n=16)$ and non-remission group $(n=29)$. Vertical lines indicate mean $\pm \mathrm{SD}$.

There were no significant differences in IgE concentrations between remission and non-remission groups.

\section{Discussion}

There are only a few reports that mention changes of TBII activities induced by pulse therapy. In one report, the author compared 2 measurements of TBII level, before and 6 months after pulse therapy, and the level decreased significantly after pulse therapy [7]. De Bellis et al. reported serial measurement of TBII levels after pulse therapy [22]. The TBII levels decreased and continued at a low level in all patients in their report. In another similar study, TBII level slightly increased in only 1 of 27 patients 12 months after pulse therapy [9]. These results gave the impression that TBII activities decrease and do not increase again in the patients who 
had received pulse therapy. Indeed, the TBII levels decreased rapidly after 3 months after pulse therapy in most patients; however, the TBII level increased again in 24 patients whose TBII was positive at 24 months after pulse therapy in our study (Fig. 2).

On the other hand, the TBII levels of the control patients decreased slowly and gradually. Decreasing rate of TBII levels after 3 months was higher in the pulse therapy group than the control group (Fig. 1, 2, $3)$. The difference in the decreasing rate is due to the immunosuppressive effect of glucocorticoids. According to these results, the immunosuppressive effect of pulse therapy followed by oral prednisolone administration continued until 6 months, but was lost from 6 months to 12 months. It is interesting that the mean TBII values at 12 months of both TBII positive groups were almost the same (Fig. 2). The pulse therapy did not improve TBII values after 12 months in the patients whose TBII was positive at 24 months after pulse therapy.

Young et al. reported that relapse of Graves' disease was not affected by the presence of ophthalmopathy [23]. On the contrary, Vitti et al. reported that the relapse rate of Graves' disease with ophthalmopathy was higher than that of Graves' disease without ophthalmopathy $(71.9 \%$ vs $57.8 \% ; p=0.08)$ [24]. Although the presence of ophthalmopathy is a possible high risk factor of relapse, it has a low predictive value alone [25]. In our study, the remission rate of Graves' disease in the 2 groups was not significantly different. The pulse therapy might increase the remission rate of Graves' disease with ophthalmopathy, but only slightly, because it did not exceed the remission rate of the control group.

The mean age of the pulse therapy group was higher than that of the control group. There is a report that indicates the remission rate is higher in older patients, possibly due to milder thyrotoxicosis, but no relationship with age was found in another study $[26,27]$. We believe that the remission rate was not affected by the difference of age between the 2 groups, because other parameters except age showed no difference. We may say from our results that pulse therapy did not contribute to the remission rate of Graves' disease. This fact also suggests that immunosuppression by pulse therapy was temporary.

Werner and Platman, and Peter reported the possible efficacy of glucocorticoid therapy for Graves' disease $[1,2]$. However, the case numbers were too small for evaluation in their studies. Mori et al. also reported the effectiveness of steroid treatment in terms of reduction in goiter size and remission in patients with Graves' disease [3]. In the patients whose TBII was negative at 24 months after pulse therapy, TBII values decreased faster than controls in our study (Fig. 2). Glucocorticoid therapy may be effective in such patients.

The degree of improvement of eyeball movement was not related to the TBII level after pulse therapy. Some patients achieved sufficient results in spite of their high TBII. Their eye signs were not exacerbated even if immunosuppressive effect was lost. The antiinflammatory effect of corticosteroids may contribute to the improvement of ophthalmopathy rather than immunosuppressive effect. These results suggest that the ophthalmopathy improves by temporary cessation of inflammation. It may be important to break the vicious circle between cytokines from lymphocytes and lymphocyte infiltration for a certain period [2830]. Autoimmune reaction may be a trigger of Graves' ophthalmopathy, however, it may not be a factor that sustains the disorder.

There was no association between prognosis of Graves' disease and IgE in this study. The reason why the result is different from past reports may be that all our subjects had active and severe ophthalmopathy, as it was suggested that $\mathrm{IgE}$ was also associated with Graves' ophthalmopathy in several reports [31-33].

In summary, our results indicated that the immunosuppressive effect of pulse therapy was temporary, and that pulse therapy did not increase the remission rate of Graves' disease.

\section{References}

1. Werner SC, Platman SR (1965) Remission of hyperthyroidism (Graves' disease) and altered pattern of serum-thyroxine binding induced by prednisone. Lancet 2: 751-755.
2. Peter SA (1991) Remission of Graves' disease with hyperthyroidism by a combination of glucocorticoids and antithyroid drugs. J Natl Med Assoc 83: 261-264.

3. Mori T, Sugawa H, Kosugi S, Ueda M, Inoue D, 
Akamizu T (1997) Effectiveness of a short-term steroid treatment on the reduction in goiter size in antithyroid drug-treated patients with Graves' disease. Endocr $J$ 44: 575-580.

4. Nagayama Y, Izumi M, Kiriyama T, Yokoyama N, Morita S, Kakezono F, Ohtakara S, Morimoto I, Okamoto S, Nagataki S (1987) Treatment of Graves' ophthalmopathy with high-dose intravenous methylprednisolone pulse therapy. Acta Endocrinol (Copenh) 116: 513-518.

5. Kendall-Taylor P, Crombie AL, Stephenson AM, Hardwick M, Hall K (1988) Intravenous methylprednisolone in the treatment of Graves' ophthalmopathy. BMJ 297: 1574-1578.

6. Marcocci C, Bartalena L, Bogazzi F, Bruno-Bossio G, Lepri A, Pinchera A (1991) Orbital radiotherapy combined with high dose systemic glucocorticoids for Graves' ophthalmopathy is more effective than radiotherapy alone: results of a prospective randomized study. J Endocrinol Invest 14: 853-860.

7. Hiromatsu Y, Tanaka K, Sato M, Kuroki T, Nonaka K, Kojima K, Nishimura H, Nishida H, Kaise N (1993) Intravenous methylprednisolone pulse therapy for Graves' ophthalmopathy. Endocr J 40: 63-72.

8. Koshiyama H, Koh T, Fujiwara K, Hayakawa K, Shimbo S, Misaki T (1994) Therapy of Graves' ophthalmopathy with intravenous high-dose steroid followed by orbital irradiation. Thyroid 4: 409-413.

9. Tagami T, Tanaka K, Sugawa $H$, Nakamura $H$, Miyoshi Y, Mori T, Nakao K (1996) High-dose intravenous steroid pulse therapy in thyroid-associated ophthalmopathy. Endocr J 43: 689-699.

10. Chang TC, Kao SC, Hsiao YL, Lu CP, Huang KM, Tzeng SS (1996) Therapeutic responses to corticosteroids in Graves' ophthalmopathy. J Formos Med Assoc 95: 833-838.

11. Matejka G, Verges B, Vaillant G, Petit JM, BrunPacaud A, Rudoni S, Brun JM (1998) Intravenous methylprednisolone pulse therapy in the treatment of Graves' ophthalmopathy. Horm Metab Res 30: 93-98.

12. Marcocci C, Bartalena L, Tanda ML, Manetti L, Dell'Unto E, Rocchi R, Barbesino G, Mazzi B, Bartolomei MP, Lepri P, Cartei F, Nardi M, Pinchera A (2001) Comparison of the effectiveness and tolerability of intravenous or oral glucocorticoids associated with orbital radiotherapy in the management of severe Graves' ophthalmopathy: results of a prospective, single-blind, randomized study. J Clin Endocrinol Metab 86: 3562-3567.

13. Macchia PE, Bagattini M, Lupoli G, Vitale M, Vitale G, Fenzi G (2001) High-dose intravenous corticosteroid therapy for Graves' ophthalmopathy. J Endocrinol Invest 24: 152-158.

14. Kauppinen-Makelin R, Karma A, Leinonen E, Loyttyniemi E, Salonen O, Sane T, Setala K, Viikari
J, Heufelder A, Valimaki M (2002) High dose intravenous methylprednisolone pulse therapy versus oral prednisone for thyroid-associated ophthalmopathy. Acta Ophthalmol Scand 80: 316-321.

15. Ohtsuka K, Sato A, Kawaguchi S, Hashimoto M, Suzuki Y (2003) Effect of steroid pulse therapy with and without orbital radiotherapy on Graves' ophthalmopathy. Am J Ophthalmol 135: 285-290.

16. Cidlowski JA, King KL, Evans-Storms RB, Montague JW, Bortner CD, Hughes FM Jr (1996) The biochemistry and molecular biology of glucocorticoid-induced apoptosis in the immune system. Recent Prog Horm Res 51: 457-490.

17. Sabir S, Werth VP (2000) Pulse glucocorticoids. Dermatol Clin 18: 437-446.

18. Sato A, Takemura Y, Yamada T, Ohtsuka H, Sakai H, Miyahara Y, Aizawa T, Terao A, Onuma S, Junen K, Kanamori A, Nakamura Y, Tejima E, Ito Y, Kamijo K (1999) A possible role of immunoglobulin $\mathrm{E}$ in patients with hyperthyroid Graves' disease. J Clin Endocrinol Metab 84: 3602-3605.

19. Yamada T, Sato A, Komiya I, Nishimori T, Ito Y, Terao A, Eto S, Tanaka Y (2000) An elevation of serum immunoglobulin $\mathrm{E}$ provides a new aspect of hyperthyroid Graves' disease. J Clin Endocrinol Metab 85: 2775-2778.

20. Komiya I, Yamada T, Sato A, Kouki T, Nishimori T, Takasu N (2001) Remission and recurrence of hyperthyroid Graves' disease during and after methimazole treatment when assessed by $\operatorname{IgE}$ and interleukin 13 . $J$ Clin Endocrinol Metab 86: 3540-3544.

21. Donaldson SS, Bagshaw MA, Kriss JP (1973) Supervoltage orbital radiotherapy for Graves' ophthalmopathy. J Clin Endocrinol Metab 37: 276-285.

22. De Bellis A, Bizzarro A, Conte M, Coronella C, Solimeno S, Perrino S, Sansone D, Guaglione M, Wall JR, Bellastella A (2003) Relationship between longitudinal behaviour of some markers of eye autoimmunity and changes in ocular findings in patients with Graves' ophthalmopathy receiving corticosteroid therapy. Clin Endocrinol (Oxf) 59: 388-395.

23. Young ET, Steel NR, Taylor JJ, Stephenson AM, Stratton A, Holcombe M, Kendall-Taylor P (1988) Prediction of remission after antithyroid drug treatment in Graves' disease. Q J Med 66: 175-189.

24. Vitti P, Rago T, Chiovato L, Pallini S, Santini F, Fiore E, Rocchi R, Martino E, Pinchera A (1997) Clinical features of patients with Graves' disease undergoing remission after antithyroid drug treatment. Thyroid 7 : 369-375.

25. Orgiazzi J, Madec AM (2002) Reduction of the risk of relapse after withdrawal of medical therapy for Graves' disease. Thyroid 12: 849-853.

26. Yamada T, Aizawa T, Koizumi Y, Komiya I, Ichikawa K, Hashizume K (1994) Age-related therapeutic 
response to antithyroid drug in patients with hyperthyroid Graves' disease. J Am Geriatr Soc 42: 513-516.

27. Schleusener H, Schwander J, Fischer C, Holle R, Holl G, Badenhoop K, Hensen J, Finke R, Bogner U, Mayr WR (1989) Prospective multicentre study on the prediction of relapse after antithyroid drug treatment in patients with Graves' disease. Acta Endocrinol (Copenh) 120: 689-701.

28. Mysliwiec J, Kretowski A, Stepien A, Kinalska I (2004) Serum levels of soluble TNFalpha receptors (sTNFR1 and sTNFR2) during corticosteroid treatment in patients with Graves' ophthalmopathy. Immunol Invest 33: 61-68.

29. Mysliwiec J, Kretowski A, Stepien A, Mironczuk K, Kinalska I (2003) Interleukin 18 and transforming growth factor betal in the serum of patients with Graves' ophthalmopathy treated with corticosteroids.
Int Immunopharmacol 3: 549-552.

30. Kumar S, Bahn RS (2003) Relative overexpression of macrophage-derived cytokines in orbital adipose tissue from patients with Graves' ophthalmopathy. J Clin Endocrinol Metab 88: 4246-4250.

31. Raikow RB, Dalbow MH, Kennerdell JS, Compher K, Machen L, Hiller W, Blendermann D (1990) Immunohistochemical evidence for IgE involvement in Graves' orbitopathy. Ophthalmology 97: 629-635.

32. Raikow RB, Tyutyunikov A, Kennerdell JS, Kazim M, Dalbow MH, Scalise D (1992) Correlation of serum immunoglobulin E elevations with clinical stages of dysthyroid orbitopathy. Ophthalmology 993: 361-365.

33. Molnar I, Horvath S, Balazs C (1996) Detectable serum IgE levels in Graves' ophthalmopathy. Eur $J$ Med Res 1: 543-546. 